\title{
CALIBRATION/VALIDATION ERROR BUDGETS, UNCERTAINTIES, TRACEABILITY AND THEIR IMPORTANCE TO IMAGING SPECTROMETRY
}

\author{
K. Thome \\ National Aeronautics and Space Administration (NASA) Goddard Space Flight Center, USA
}

\begin{abstract}
Knowledge of uncertainties and errors are essential for comparisons of remote sensing data across time, space, and spectral domains. Vicarious radiometric calibration is used to demonstrate the need for uncertainty knowledge and to provide an example error budget. The sample error budget serves as an example of the questions and issues that need to be addressed by the calibration/validation community as accuracy requirements for imaging spectroscopy data will continue to become more stringent in the future. Error budgets will also be critical to ensure consistency between the range of imaging spectrometers expected to be launched in the next five years.
\end{abstract}

Index Terms - Calibration, validation, imaging spectroscopy, hyperspectral, traceability, error budget

\section{INTRODUCTION}

An important aspect of the use of imaging spectrometer data, and all remote sensing data for that matter, is the characterization and calibration of the sensors and validation of their data products. Just as important is the development of demonstrated error budgets to determine the accuracy and precision of the sensor characterization and ultimately the data products. There exist numerous methods for accomplishing the pre-flight and on-orbit characterization of sensors. Likewise, there is a range of uncertainties that are required that depend primarily on the application of interest. The current work does not describe how these requirements are determined or the values that should be achieved. Rather, the purpose of this work is to convince the reader that undertaking the development of a traceable and defensible error budget will improve their own data with the added benefit that other groups will benefit as well.

The current work concentrates on the development of error budgets for radiometric calibration the concepts presented here are valid across a variety of other sensors and quantities. Developing error budgets that maintain a traceability to an agreed-upon standard can remove biases between data sets from different sensors operating at the same time or similar sensors being used to develop a time series. Providing a traceable error budget for radiometric calibration also ensures band-to-band consistency across the full spectral range of the large number of bands encountered in imaging spectroscopy.

\section{TERMINOLOGY}

One of the first things any researcher finds when attempting to determine an error budget for their work is the wide array of terms that can be used and the even wider array of possibly meanings for those terms. Many of the terms have seemingly inconsistent definitions depending on the organization or metrology laboratory providing them. The current work attempts to avoid confusion and controversy by using three broadly understood (and misunderstood) terms: traceability, accuracy, and precision. It is understood that there are more precise terms that can more accurately describe the calibration/validation process (such as Type A and $\mathrm{B}$ uncertainties). Discussion of terminology definition is left for a lengthier treatise. One reason for taking what appears to be a cavalier attitude towards terminology is that the specifics of terms are still evolving within remote sensing and this is especially true due to the relatively recent inclusion of national metrology institutes in traceability and uncertainty evaluations $[3,4]$.

Precision in this work is taken to be equivalent to repeatability and accuracy is meant to imply the difference between a given result and the actual value. Both repeatability and actual value have their own inherent definition problems, but again, the goal is to discuss the need for uncertainty and error budgets, not specific nomenclature. In addition, the discussion here uses uncertainty in place of accuracy so that the quantitative values for both the precision and the accuracy are similar. That is, a $3 \%$ precision implies $97 \%$ accuracy and uncertainty would be $3 \%$.

Traceability refers to adherence to an agreed upon standard. Again, there is specific guidance on how traceability should 
be achieved (Guidelines for Evaluating and Expressing the Uncertainty of NIST Measurement Results [5]) but only the concept of traceability is needed for this discussion. The most basic view of traceability is that an error budget determines the uncertainty relative to the agreed-upon standard. SI-traceability is achieved if that standard is part of the Systeme International. The advantage of developing a truly traceable error budget is that the same physical measurement from different sensors will be directly comparable if the uncertainties are traced to the same standard.

\section{NEED FOR ERROR BUDGETS}

The ultimate goals for radiometric calibration are to place the data on scale that allows for consistent data product output from the sensor of interest. Calibrated data products remove striping present in a single band of a given sensor and biases between spectral bands. Temporal degradation can be evaluated and removed. Placing the absolute calibration on an agreed-upon scale allows results from multiple sensors to be compared while minimizing the impact of sensor-related artifacts.

Figure 1 illustrates the importance of knowing the uncertainty of the sensor's characterization in assessing the temporal degradation. The results are for the Advanced Land Imager that is a multispectral, pushbroom sensor with $30-\mathrm{m}$ spatial resolution and give the percent difference between the reported spectral radiance from several of the bands of ALI and those predicted based on ground measurements at the time of ALI overpass [1]. The bands shown are approximately centered at 450, 550, 650, 850, 1600 , and $2200 \mathrm{~nm}$. Negative percent differences indicate that sensor reported radiances are less than predicted values based on in situ ground values. The error bar shown in the graph is a crude approximation derived from the standard deviation of the average percent difference but still serves to illustrate that knowledge of the uncertainty is needed in order to determine whether degradation has occurred or not. In this case, there is no statistically significant degradation seen in the ALI sensor.

Such ground data can be used to determine whether biases exist between two different sensors. Figure 2 illustrates this between the Enhanced Thematic Mapper Plus (ETM+) and ALI that are on two separate platforms but in similar orbits that were within minutes of one another for the comparison shown here. Here again, the uncertainty bar shown for each band is a crude estimation based on the standard deviation of the average percent difference.
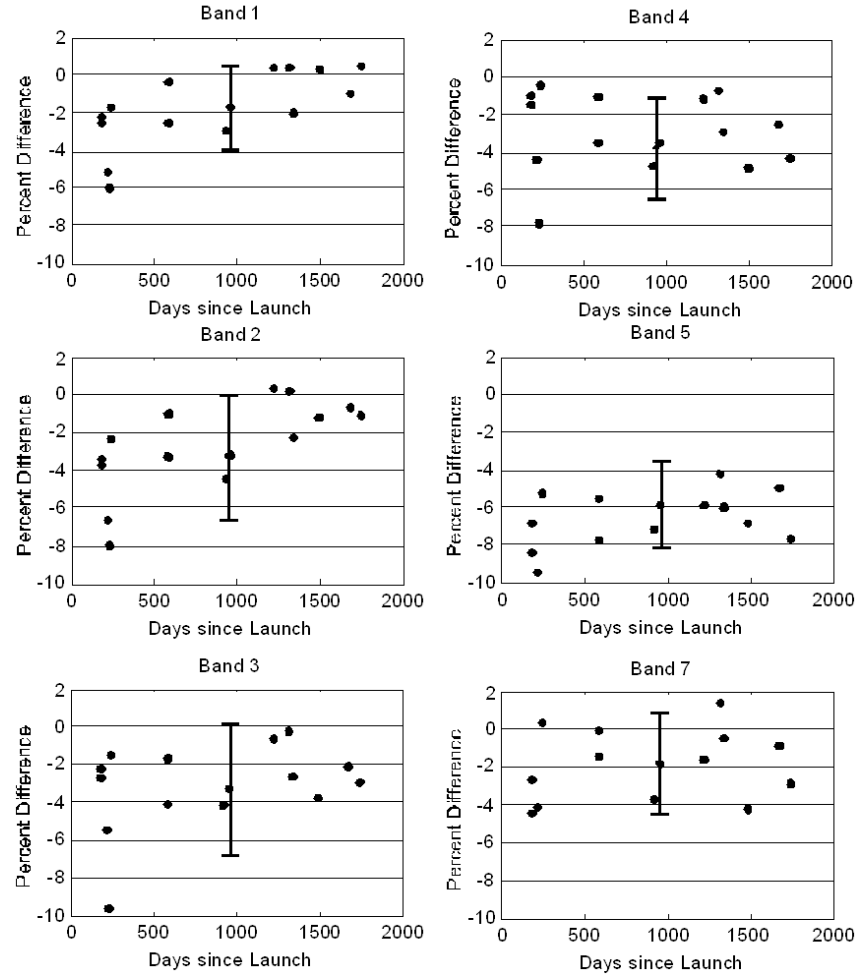

Figure 1: Percent difference between vicarious predictions and reported sensor radiance for ALI as a function of time since launch. Also shown is the $1-\sigma$ standard deviation of the average percent difference of all data points.

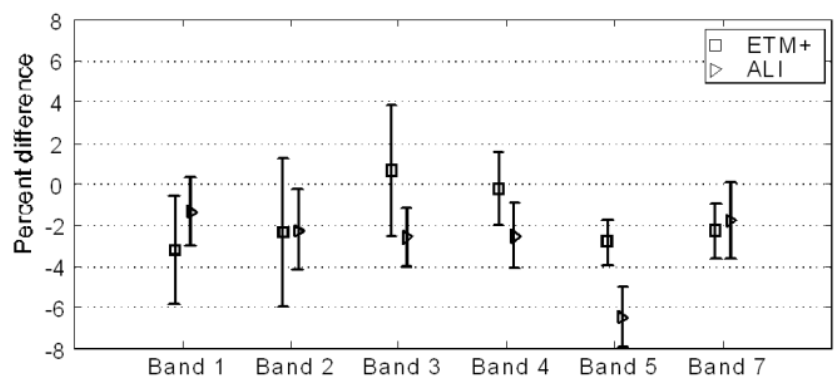

Figure 2: Average percent difference and standard deviation between reflectance-based predictions and reported sensor radiance computed for ETM+ and ALI.

The above approach is also applicable to imaging spectrometers as demonstrated by Figure 3 that shows an equivalent plot as Fig. 2 but for Hyperion (an imaging spectrometer on the same platform as ALI and with similar spatial resolution) [2]. In all cases, knowledge of an uncertainty of some kind is needed to allow inference regarding changes in time or biases. 

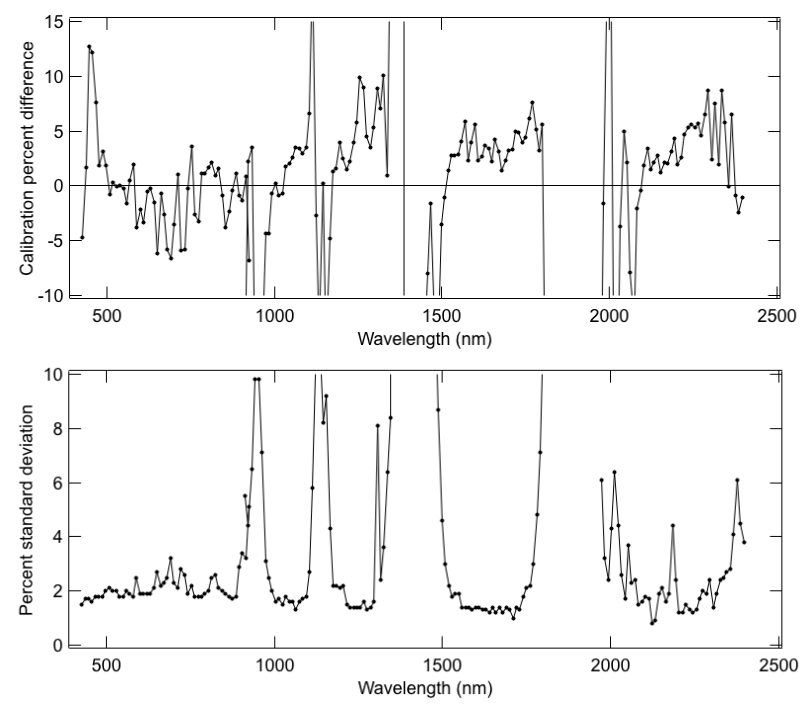

Figure 3: Average percent difference (top) and standard deviation (bottom) for vicarious results of Hyperion.

\section{EXAMPLE ERROR BUDGET}

The results shown above are based on relying upon the standard deviation as being representative of the methods uncertainty. Such an approach can be acceptable for some applications, but it does not provide traceability or an absolute uncertainty/accuracy. Future imaging spectroscopy missions will have to develop more rigorous and detailed error budgets for their on orbit calibration and validation. A vicarious calibration relying on surface reflectance and atmospheric measurements is provided here [6] as an example of the type of analysis that will be needed for future missions. Details of the error budget are not provided due to space limitations, but there should be sufficient information to provide the reader with a starting point towards developing their own traceable error budget. It should be noted that the error budget discussed here is currently undergoing its own evaluation with the help of several metrology laboratories to improve the rigor and traceability of the uncertainties.

The vicarious method relies on a set of representative input parameters: 1) sun-sensor geometry; 2) molecular optical depth; 3) aerosol optical depth; 4) column ozone; 5) column water vapor; 6) Junge parameter that defines the aerosol size distribution; 7) real index of refraction for the aerosols; 8) imaginary index of refraction for the aerosols; and 9) surface reflectance. Inputs can be parameterized in a different manner, but the basic premise is the same - inputs are related to surface and atmospheric properties. The error budget process then requires understanding the uncertainty that can be associated with determining the input parameters and the impact of that uncertainty in determining a top-ofatmosphere (TOA) radiance used for the vicarious calibration to obtain Table 3.

\begin{tabular}{|c|c|c|}
\hline Source & Source & $\overline{\text { TOA }}$ \\
\hline Ground reflectance measurement & & 2.2 \\
\hline $\begin{array}{l}\text { Reference panel } \\
\text { reflectance factor }(\mathrm{BRF}) \text { calibration }\end{array}$ & 2.0 & \\
\hline Diffuse-field correction & -- & \\
\hline Measurement errors & 1.0 & \\
\hline Optical depth measurements & 0.5 & $<0.1$ \\
\hline Extinction optical depth & 0.5 & \\
\hline \begin{tabular}{|l} 
Partition into Mie and Rayleigh \\
\end{tabular} & -- & \\
\hline Absorption computations & & --- \\
\hline Column ozone & 2.0 & \\
\hline Column water vapor & 5.0 & \\
\hline Choice of aerosol complex index & 100 & 0.5 \\
\hline Choice of aerosol size distribution & --- & 0.3 \\
\hline Type & -- & \\
\hline Size limits & -- & \\
\hline Junge parameter & 0.3 & \\
\hline Non-lambertian ground characteristics & 10 & -- \\
\hline Other & & \\
\hline Vertical distribution & & -- \\
\hline Non-polarization versus polarization & & 0.1 \\
\hline Inherent code accuracy & & 1.0 \\
\hline Uncertainty in solar zenith angle & & 0.2 \\
\hline \begin{tabular}{|l} 
TOTAL ROOT \\
(RSS) ERROR
\end{tabular} & & 2.5 \\
\hline
\end{tabular}

Table 3. Error assessment developed for vicarious calibration of visible spectral bands. All values in the table are percentage values. Those in the "Source error" column list the percent error in the determination of that parameter. Values in the "TOA error" column are the percent changes in the TOA radiance due to the source errors.

A variety of approaches were used to determine the values in Table 3. Average values for aerosol parameters were found and their variability used to derive an uncertainty. Standard deviations are somewhat flawed in that several parameters are physically limited to values greater than zero, and have frequency distributions skewed towards lower values, but the variability of the averages has been found to follow expected accuracy of the instruments as given by other sources. Still, such an approach of using the variability does not strictly determine the accuracy of the input but rather provides an estimate of site variability and the TOA radiance sensitivity to that variability. The use of the input variability has been helpful in determining which part of the vicarious calibration would benefit from improved measurements, but the current collaborations with metrology laboratories should lead to a more rigorous and traceable uncertainty. 
Many of the input parameters are determined through assumptions about the conditions at the test site, climatological values, or selections that make the processing methodology simpler. Assessing the impact of these choices is done through a sensitivity study similar to that described in the previous paragraph. The reliance on assumed values does not strongly impact the TOA radiance, hence the original choice to use assumed values.

The greatest rigor in assessing an absolute uncertainty was applied to the determination of the surface reflectance. Assessment of the impact of assuming that hemisphericconical reflectance factor measurements can be used to derive bi-directional showed this to be negligible due to high surface reflectance and low aerosol loading. The sites were assessed relative to show that near-nadir views were not impacted by assuming a lambertian surface. That is, the uncertainty caused by non-lambertian behaviour could not be separated from other variabilities.

The dominant sources of error in the retrieval of surface reflectance are panel reference calibration, field spectrometer temporal stability, exclusion of diffuse-light correction, and sampling issues related to measuring the test site and registering these measurements to the sensor being calibrated. The error due to the reference is labeled as the "panel bi-directional reflectance (BRF)" entry in the table. All of the other factors besides the diffuse-light factor are included in measurement errors. The $2 \%$ value in the table is based on round-robin results and detailed uncertainty analysis of the laboratory set up. Studies of the field spectrometer show that it can cause variations in the retrieved reflectance on the order of $1 \%$ in the mid-visible and evaluation of the diffuse-light effect shows it to be negligible for typical conditions for wavelengths longer than $500 \mathrm{~nm}$. Sampling uncertainties were shown to be negligible for sensors with footprints $<50 \mathrm{~m}$.

A benefit of an error budget study is that it showed that impacts from assumptions of the aerosol size distribution and its parameterization are not a large source of uncertainty for a high-reflectance surface with low aerosol loading. The error budget development led to studies evaluating multiple aerosol parameterizations, and measurement approaches. It was this work that led to the entry in the table related to choice of distribution being negligible, as is the entry related to the minimum and maximum.

Taking the root sum square (RSS) of the tabular values leads to an absolute uncertainty for this vicarious approach of $2.5 \%$. This is for the mid-visible to red part of the spectrum for a typical, clear-sky day. Comparison with sensors such as ALI and ETM+ indicate that the derived uncertainty is not wildly incorrect. The traceability in this case is to a bi- directional reflectance standard developed by the National Institute of Standards and Technology.

As important as absolute accuracy is to understanding the absolute radiometric accuracy of a sensor, the precision of the vicarious method plays a role in allowing multiple sensors to be compared as in Figure 2. Monte Carlo simulations of TOA radiance predictions using typical input parameters and uncertainties show that the standard deviation of the average indicates a reasonable approximation of the methods precision. Such an approach is far from rigorous but allowed early conclusions to be drawn regarding sensor harmonization. The current efforts to improve the error budget will provide a much better precision estimate.

\section{CONCLUSIONS}

The goal of this work is to present a beginning error budget development approach that imaging spectroscopists could apply to the calibration and validation of their data. It is difficult to provide a detailed approach within the current work's page limitations, but the hope is that readers will start such a process with the realization that periodic iterations and peer review of their results will lead to more accurate accuracy assessments.

\section{REFERENCES}

1] J. McCorkel, K. J. Thome, S. Biggar, and M. Kuester. "Radiometric calibration of Advanced Land Imager using reflectance-based results between 2001-2005,." Proceedings of SPIE Vol. 6296, 2006.

[2] McCorkel, J., K. J. Thome, and L. Ong. "Vicarious Calibration of EO-1 Hyperion." IEEE J. Sel. Top. Appl. Earth Observations Remote Sensing, Vol. 6, pp 400-407, 2013.

[3] K. J. Thome, S. Schiller, J. Conel, K. Arai, and S. Tsuchida. "Results of the 1996 Earth Observing System vicarious calibration campaign at Lunar Lake Playa, Nevada (USA)." Metrologia, Vol. 35, pp. 631-638, 1998.

[4] K. J. Thome and N. Fox. "2010 CEOS field reflectance intercomparisons lessons learned.", Geoscience and Remote Sensing Symposium (IGARSS)2011 IEEE International, Vancouver, BC. pp. 3879-3883, 2011

[5] B. N. Taylor, C. E. Kuyatt, "Guidelines for Evaluating and Expressing the Uncertainty of NIST Measurement Results." NIST TN 1297, 20 pp., 1994.

[6] K. J. Thome, C. Catrall, J. DAmico, and J. Geis. "Ground-reference calibration results for Landsat-7 ETM+." Proceedings of SPIE 5882, 2005. 